\title{
Nueva Galicia a través de la historiografia regional
}

\author{
Beatriz Rojas \\ INSTITUTO MORA
}

$\mathrm{E}$ n los últimos 25 años ha crecido en la historiográfia mexicanista la producción de trabajos de carácter regional; aunque creo que la historia regional no ha terminado su labor, una rápida revisión permite ver que los avances han sido considerables.

Los investigadores que se han dedicado a hacer "historia regional" se pueden agrupar en diversas corrientes metodológicas, temáticas y analíticas. Dentro de este proceso de construcción de la historia regional, quiero destacar la reflexión sobre el origen y la existencia de las regiones. Tomaré el caso de Nueva Galicia y su capital Guadalajara, pero me limitaré a los trabajos dedicados al periodo colonial, por considerar que fue durante éste que la gran región fijó su personalidad, sin que esto signifique la negación del influjo y de la importancia que tuvieron las circunstancias del periodo prehispánico.

NUEVA GALICIA

Hasta fechas recientes los investigadores avalaron y aceptaron la existencia de Nueva Galicia como una unidad encabezada por Guadalajara, la capital del reino. Los cuestionamientos surgieron cuando, al avanzar en los estudios de carácter regional, empezaron a distinguirse diversas modalidades de desarrollo dentro del amplio territorio que conformó Nueva Galicia. Esto se debió por un lado a las evidentes dife. rencias regionales, pero también a las diversas perspectivas de análisis que existen dentro de los estudios de este tipo. El investigador que buscaba la lógica de la organización económica en este amplio territorio, tuvo que aceptar que no se trataba de una región económicamente unificada, sino que estaba compuesta por diversas regiones que obedecían, en su organización interna, a circunstancias múltiples que las diferenciaban entre sí.

Pero entonces: ¿cómo explicar la evidencia de que Nueva Galicia había formado un todo? y ¿qué explicación dar a una congruencia regional evidente en muchos aspectos? Fue así como varios investigadores se dieron como tarea, no explicar las diferencias regionales, sino, al contrario, bus- 
car los elementos que conformaban la unidad regional.

Dentro de esta tendencia destacaron por su temprana aparición los trabajos de dos historiadores franceses: el de Jean Pierre Berthe titulado Introduction al'histoire de Guadalajara et sa region y el de Jean Meyer Perspectives de l'analyse socio-historique de l'influence de Guadalajara sur sa région, ambos elaborados en 1967, dentro del marco de un programa de investigación impulsado por el Centre National de la Recherche Scientifique (CNRS). Ese proyecto culminó con la publicación de dos trabajos pioneros: Villes et régiones en Amérique Latine, Cahier, núm. 1, Institut des Hautes Etudes de Amérique Latine, 1970, y la tesis de Hélene Riviere d'Arc, Guadalajara et sa région. Influences et difficultés d'une métropole mexicaine, que apareció en Francia en 1970 y en México en 1973 en la colección SepSetentas.

Quiero destacar en mi reflexión el trabajo de J. P. Berthe, porque aborda directamente la validación histórica de la existencia de Nueva Galicia como una unidad y la relación establecida entre la ciudad y la region. Los lineamientos que fijó en este artículo han nutrido durante los últimos 25 años las investigaciones que tratan este asunto.

El profesor Berthe presenta como hecho determinante en la formación de Nueva Galicia, primero la constitución de una Audiencia y posterior: mente la concentración en Guadalajara del poder eclesiástico y de las funciones administrativas y judiciales. ${ }^{1}$ Para

${ }^{1}$ Berthe, "Introduction", 1970, p. 70. Para él, el territorio de este reino fue en gran medida definido por los límites de la jurisdicción de la Audiencia: aclara que este hecho no fue un privilegio de Nueva Galicia, sino que se reprodujo en otras partes del imperio español. La dependencia de un territorio en relación con un centro administrativo terminó por crear relaciones privilegiadas. Este presupuesto lo avala con la opinión de Matías de la Mota Padilla quien en 1742 señaló cómo Guadalajara fue la "ciudad patria común a cuantos están avecindados en el reino."2

Berthe reconoce que el elemento de dependencia administrativa no es suficiente y señala que otras variables tomaron parte en la definición de esta entidad, pero ninguna le parece tan evidente en la creación de la "conciencia regional" como la ligada al centro administrativo. Por lo mismo no se puede subestimar la importancia de la continua batalla librada entre las autoridades novohispanas y las neogallegas, para defender lo que cada una de ellas consideraba, dependía de su autoridad. En esa lucha para defender su ámbito de poder, Nueva Galicia consolidó su personalidad.

Entre las variables a tomar en cuenta J. P. Berthe señala también la importancia del tipo de poblamiento existente en este reino "donde domina el elemento no indio". 3

ver con detenimiento todo el proceso de organización de las instituciones de gobierno políticas y eclesiásticas durante el siglo XVI, ver el trabajo de Parry apenas publicado en español, Audiencia, 1993.

2 Citado por el profesor Berthe, "Introduction", 1970, p. 70.

${ }^{3}$ No podemos extendernos en las variables que Berthe distingue. Sin embargo es importan- 


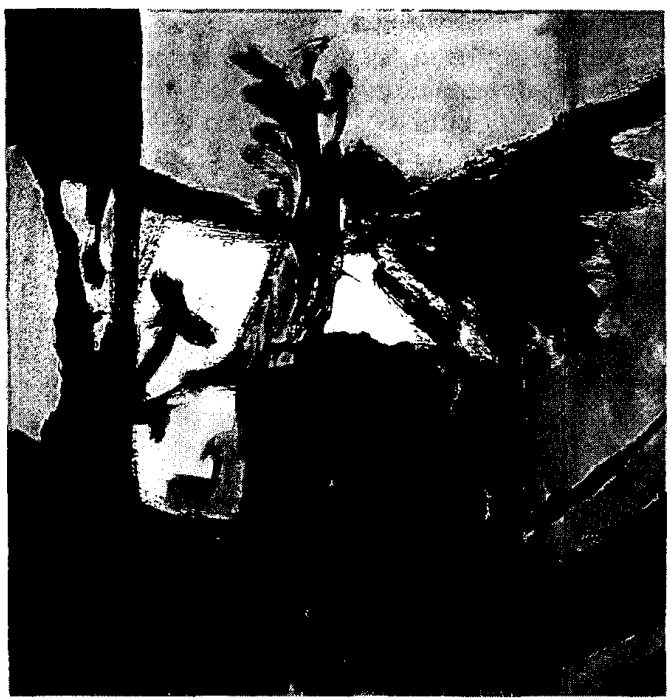

Distingue implícitamente varias etapas en la formación de esta conciencia. Los primeros pasos se dieron involuntariamente puesto que ni siquiera la sede de la Audiencia fue escogida premeditadamente, por lo cual tuvo que cambiar de lugar en más de una ocasión. Después vino la cristalización de las fuerzas regionales, etapa que llegó cuando su capacidad económica les permitió existir por sí mismas, lo que sucedió en los últimos años del siglo XVIII. Este proceso no se consoli-

te señalar que encuentran concordancia con las propuestas de otros estudios como sería el ya clásico trabajo coordinado por Altman y Lockhart, Provinces, 1976. El trabajo de J. Meyer trata más directamente sobre los condicionantes sociales que dan unidad y coherencia al territorio de Nueva Galicia. Ver passim. dó sino hasta que se tomó conciencia de un sentimiento regional que se transformó en demandas y reivindicaciones para consolidarlo, en el último cuarto del siglo Xvis. El proceso culminó con la afirmación de una clara y articulada conciencia regional en el Memorial Petitorio de 1817, cuando la dependencia con la capital del virreinato engendró un sentimiento de "opresión". J. P. Berthe concluye diciendo que:

La toma de conciencia regional se debió a la conjunción de un desarrollo económico muy marcado durante el último cuarto del siglo XVII en las estructuras agrícolas y comerciales, con una tradición antigua de particularismo administrativo. ${ }^{4}$

${ }^{4}$ Berthe, "Introduction", p. 74. 
Y señala cómo durante el último cuarto del siglo XVIII "se afirma, en efecto, un determinado número de caracteres de la región y de la ciudad" 5 promovidos y afianzados con la creación de la universidad, la instalación de la imprenta, pero sobre todo con la creación del Consulado. Berthe encuentra que después de 1750 una serie de factores consolidan la región: la apertura del puerto de San Blas en 1770 que abre el conducto comercial con Sonora y las Californias, el mejoramiento de caminos, el reforzamiento de la feria de San Juan, la construcción de puentes. Contribuye al desarrollo comercial la interrupción del comercio interoceánico por la guerra europea que favoreció también el desarrollo económico del reino, sobre todo en el ramo de los textiles.

J. P. Berthe, señala que su trabajo no aborda todas las variables que participaron en el "nacimiento de la solidaridad regional en torno a Guadalajara". Sin embargo demuestra claramente, que el afianzamiento de la "solidaridad o conciencia regional" se desarrolló en la coyuntura económica de finales del siglo XVIII que vivió toda Nueva España, pero también en el marco de la nueva política de la corona española.

Para corroborar lo que dice Berthe, disponemos de los testimonios de Matías de la Mota Padilla en su Historia del reino de la Nueva Galicia en la América septentrional, los cuales sin duda reflejan la forma de pensar de sus contemporáneos, de las autoridades y de los grupos de intereses neogallegos, mostrándonos cómo las elites

\footnotetext{
${ }^{5}$ Ibid., p. 73.
}

neogallegas eran sensibles a las diferencias existentes entre los dos reinos.

De la Mota Padilla se queja abiertamente de las desventajas que vivía Nueva Galicia frente a Nueva España, lo que limitaba su riqueza y desarrollo. Señala cómo México se beneficiaba con los productos y con las rentas de Nueva Galicia, con la recaudación de las alcabalas, los productos de la casa de moneda por las platas y monedas acuñadas, las rentas de pólvora, tabacos, barajas, gallos, alumbre. ${ }^{6}$ Reivindica el que muchos de los indios de Galicia no paguen tributos por lo cual sus recaudaciones disminuyen y antes bien reciben ayuda para su sustento y vestido, gastos que Nueva España no soportaba, por lo cual tenía más entradas en el ramo de tributos. En síntesis dice:

si se diera fomento al reino de Galicia, con el comercio de Filipinas en los puertos que tiene en las costas del sur, si se pusiera una casa de moneda y si no necesitaran todo de ocurrir a México, en donde todas la utilidades se epilogan [...] porque en la realidad, a estos reinos de Vizcaya y Galicia se les tienen cortadas las alas y todo el nombre de América septentrional, que a la verdad, es de un nuevo mundo, siendo tan dilatado, se ha reducido a la grandeza de un México, que está como en la puerta, y en ella se detiene cuanto de la Europa pasa, y cuanto de las Filipinas se comercia, y a esta puerta se ven precisados a ocurrir todos los que habitan el centro de dicho reino y no pueden dedicarse al cultivo de la tierra, al beneficio de los metales y a la conversión de

${ }^{6}$ Mota, Historia, 1973, p. 319. 
los infieles, por los costos, dilaciones y riesgos; y dividiendo las intendencias, pueden en pocos años Galicia y Vizcaya competir con el resto de Nueva Espaกัa...?

Cuando a finales de siglo XviII Nueva Galicia obtuvo su universidad, su consulado, después de la apertura del puerto de San Blas, tenía años de reclamarlos: eso se logró por que sus demandas coincidieron en ese preciso momento con la política del gobierno.

\section{LOS LINEAMIFNTOS DE BERTHE} 25 AÑOS DESPUÉS

Leer el trabajo del profesor Berthe a 25 años de su aparición resulta de gran interés, porque nos damos cuenta de cómo las lineas de investigación abiertas por él se han ido desarrollando paulatinamente. Eso se percibe en los trabajos de Ramón Serrera, de Thomas Calvo, de Eric van Young y también en los de Carmen Castañeda, Linda Greenow y Richard Lindley. ${ }^{8}$ En fecha más reciente, en los de Jaime Olveda, de Brian Connaughton y de María Ángeles Gálvez Ruiz. ${ }^{9}$ La característica que comparten estos trabajos, consiste en que, aunque abordan temas, periodos y regiones diferentes, todos

${ }^{7}$ Ibtd., p. 320.

${ }^{8}$ Serrera, Guadalajara, 1977; Van Young, Ciudad, 1986; Calvo, Poder, 1991; Lindley, Haciendas, 1987; Greenow, Spatial, 1980y Credit, 1983. Tan sólo el de Van Young por el tipo de análisis que realiza se aleja de esta tónica, ya que se circunscribe a delimitar el área de influencia económica de la ciudad de Guadalajara.

9 Olveda, Oligarquía, 1991; Connaughton, Ideología, 1992; Gálvez, Conciencia, 1996. buscan precisar las variables que participaron en la conformación de Nueva Galicia.

Cada uno de los autores citados, delimita el espacio que estudia en función de su concepto de región o de su análisis, con el deseo, explícito o no, de encontrar lazos de unión. José Ramón Serrera define el territorio que estudia, con criterios "esencialmente históricos en el que también se han tenido en cuenta los factores geográficos, culturales, económicos y administrativos". ${ }^{10}$ Pero, para definir la región que demarcó su estudio, tomó en cuenta dos factores primordiales: la madurez administrativa y la jurisdicción fiscal. Señala cómo Nueva Galicia vivió un proceso de madurez administrativa, en donde:

Las fronteras se van modificando progresivamente en esta centuria [siglo XVIII], incorporando algunos distritos y desprendiéndose de otros, hasta lograr a fines de siglo unos límites que ya se ajustan mejor a las necesidades administrativas y al poder de control de la capital del territorio. Incluso las fronteras que estableció en la Intendencia la Ordenanza de 1786 fueron alteradas en varias ocasiones hasta que se logró el equilibrio y la coherencia regional deseada. ${ }^{11}$

En su análisis Serrera concluye en el predominio del factor administrativo, en la formación y coherencia del reino. Igual hace Thomas Calvo cuando dice que en la estructuración del territorio neogallego fueron determinan-

${ }^{10}$ Serrera, Guadalajara, 1977, p. 2.

11 Ibid., p. 4. 


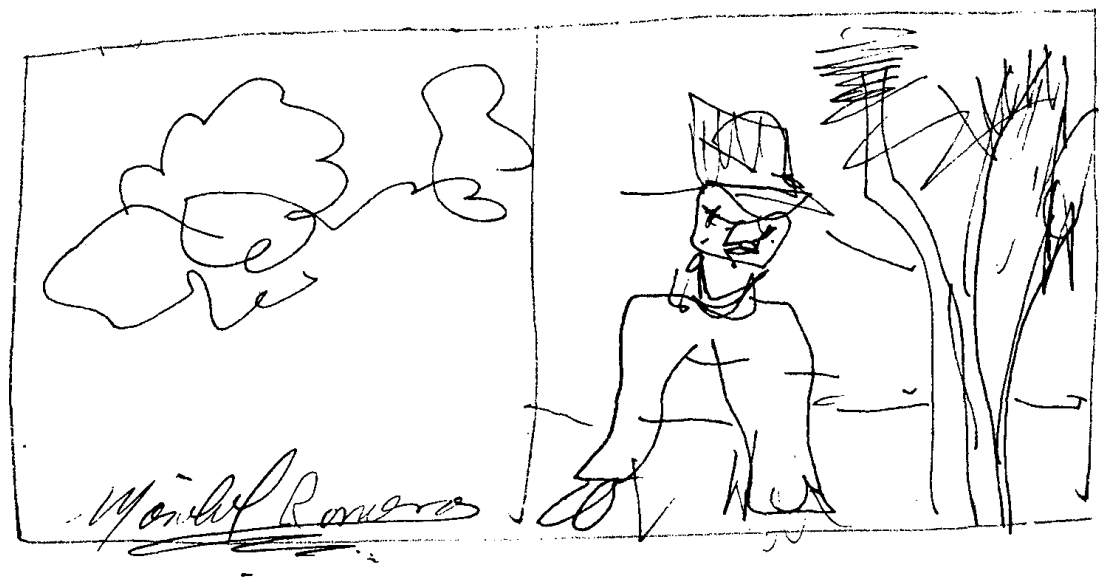

tes las funciones de la Audiencia, a la que califica de "poder que estructura el conjunto" y señala cómo: "En ningún dominio, ni demográfico, ni económico, la unidad regional aparece tan claramente como a través del trabajo rutinario de la Audiencia". ${ }^{12}$

Conforme avanzan las investigaciones, los historiadores han detectado nuevos elementos para explicar la cohesión del territorio neogallego. Linda Greenow encontró en el crédito un elemento "confederador" en el sentido de que creaba lazos de unión, término usado también por Calvo al referirse a la función desarrollada por el comercio. A estas tesis se une Lindley, al estudiar la elite de la ciudad de Gua-

12 Calvo, "Réalites", 1993. dalajara, en quien encuentra la cabeza rectora de los intereses neogallegos, representados económicamente en el dominio de la tierra, en el control del comercio y en el manejo del crédito. Toma en cuenta los lazos que cohesionan la elite, los de parentesco. Como también lo hacen Thomas Calvo y Carmen Castañeda.

Lindley se concreta a estudiar la elite de Guadalajara y a delimitar su área de influencia a lo que él considera como las fronteras de la economía "local", entendida como la zona de origen de los productos que abastecían el mercado de Guadalajara. Define el espacio de Guadalajara por las haciendas que poseía la elite urbana de Guadalajara. Así, considera que Lagos, Aguascalientes, León, Valladolid, 


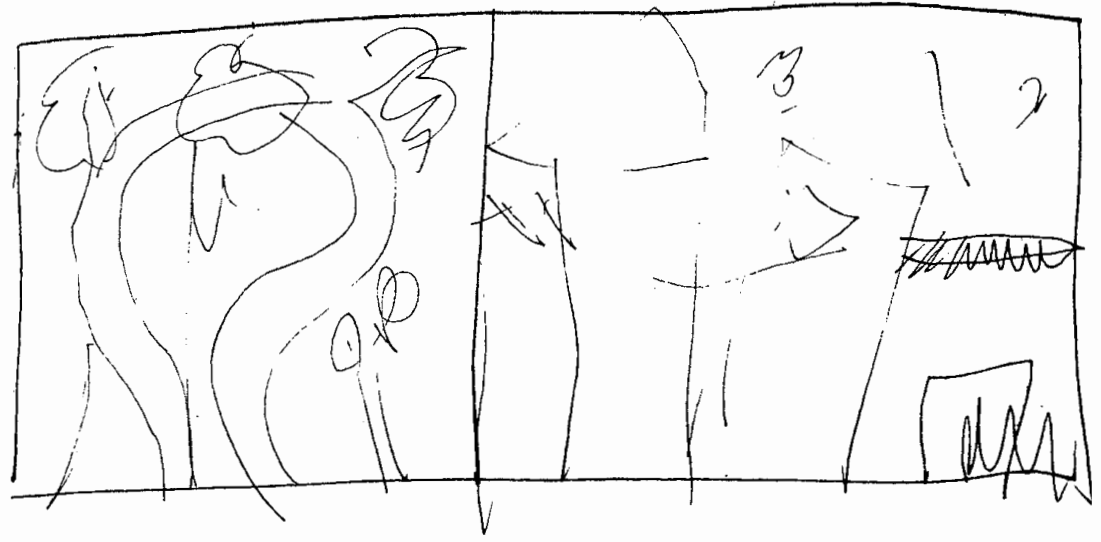

Zapotlán, Tepic y Zacatecas, estaban fuera de la influencia de esta elite. ${ }^{13}$ Esto lo señalo porque, como veremos más adelante, conforta parcialmente las propuestas de Jaime Olveda.

Los trabajos de Olveda, Connaughton y Gálvez, de reciente aparición, siguen las pistas abiertas por Berthe $y$ aunque sus trabajos tienen temas diferentes, los tres se preocupan por definir lo que consideran la unidad neogallega y el área de influencia de Guadalajara.

Los tres trabajos abordan principalmente el periodo tardío del siglo XviII, momento preciso que todos los autores señalados consideran como el decisivo en la consolidación de la concien-

${ }^{13}$ Lindley, Haciendas, 1987, p. 56. cia regional. Olveda y Connaughton prosiguen sus análisis hasta mediados de siglo XIX, y hacen un corte cronológico más acorde con las periodiza. ciones propuestas últimamente por los historiadores que tienen estas preocupaciones. En la lectura de estos trabajos hay que tomar en cuenta los cambios que vivió Nueva Galicia al aplicarse las Ordenanzas en 1786. La constitución de las intendencias transformó sustancialmente la constitución del antiguo espacio neogallego, al desarticular la unidad política del antiguo reino: sólo se mantuvo la unidad judicial. No entraré en más detalles sobre este asunto, porque aunque estos cambios fueron importantes desde su aplicación en 1787, se volvieron determinantes con la aplicación de la Constitu- 
ción de Cádiz en 1812. Pero, veamos lo que dicen nuestros autores.

Olveda da a los conquistadores de este reino, un papel determinante en la constitución de la unidad regional. Piensa que las regiones se modelaron a su arbitrio y al de sus descendientes en función de su propiedad territorial, "de esta forma las regiones se fueron configurando y organizando de acuerdo a sus intereses". ${ }^{14}$ Describe la génesis de la oligarquía terrateniente neogallega, dueña, gracias a las mercedes de tierra que obtuvo, de gran parte del territorio adyacente a Guadalajara.

Pero esta oligarquía neogallega, conformada por los principales terratenientes criollos y por los comerciantes peninsulares, grupos que estaban unidos por lazos familiares y por intereses económicos, no logró consolidar su poder sino hasta que la liberalización del comercio le permitió expandir su dominio. Dice Olveda que el desarrollo que vivió Guadalajara en los últimos años del siglo XVIII, reforzó el sentimiento regional de las elites, "las cuales actuaron cada vez con mayor decisión, reclamando más libertad, primero económica y posteriormente política". 15

Gracias a las reformas borbónicas, las oligarquías regionales "se fortalecieron y tomaron conciencia de la capacidad que tenían para vivir autónomamente". Lo que llevó a la elite de Guadalajara a plantear a la corona un conjunto de demandas "para dotar a la ciudad de todas aquellas instituciones que pudieran fortalecerla como capi-

14 Olveda, Oligarquía, 1991, p. 21.

15 Ibid., p. 76. tal económica, política, administrativa y cultural". Fue durante este periodo que la ciudad

reafirmó su función de centro redistribuidor de productos importados; se establecieron instituciones que fundaron la conciencia regional, y se registró una mayor contradicción social a consecuencia del ensanchamiento de la brecha que separaba a la oligarquía de los grupos subalternos.

El trabajo de Ma. Ángeles Gálvez Ruiz que lleva por título La conciencia regional en Guadalajara y el gobierno de los intendentes 1786-1800 está construido en torno a la ciudad de Guadalajara y busca rastrear la afirmación de la conciencia regional. Las tesis que maneja son las mismas que las de otros autores, pero ella se explaya más porque efectúa una minuciosa revisión de cómo y por quiénes se llevó a cabo este proceso, aprovechando extensamente los trabajos que se han realizado en las últimas décadas y la exhaustiva consulta de archivos. Su análisis se centra en las reformas político-administrativas de los últimos tres decenios del siglo xviII. Visto en conjunto, en este trabajo sobresalen como explicación de la consolidación de la "conciencia regional" los conflictos surgidos por la redistribución del poder ligada a la aplicación de las Ordenanzas. La función del intendente en este proceso queda especialmente señalada por una doble tarea: confirmar a Guadalajara en su función de capital del reino de Nueva Galicia y consolidar la autonomía de su poder frente a los virreyes. En este proceso, 


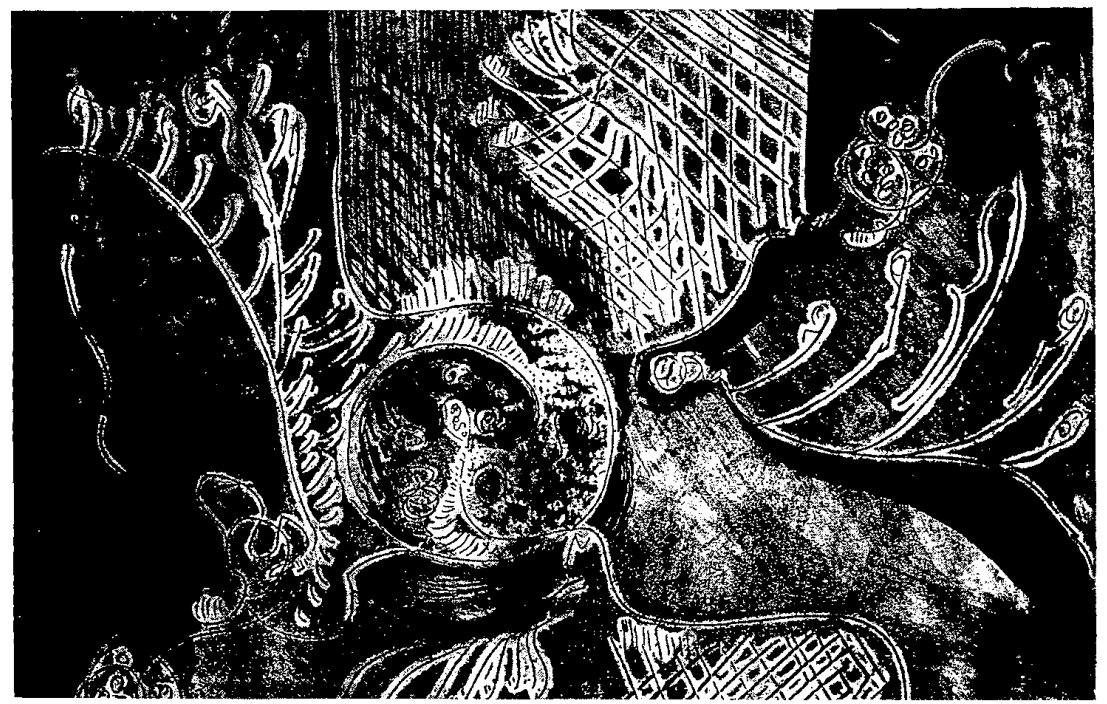

señala la autora, la actitud del intendente Jacobo Ugarte fue fundamental pues "se presenta como un personaje reivindicativo y representante máximo de la conciencia regional" ${ }^{16}$

Incluso quiso llevar su proyecto hasta la constitución de un nuevo virreinato, pero fue desarticulado por la oposición del virrey Branciforte quien recibió el apoyo del príncipe de la paz. ${ }^{17}$

Para Gálvez, no fue tan sólo el poder civil el que se empeñó en consolidar este sentimiento. Los dos obispos que tuvo esta diócesis en este periodo, fray Antonio Alcalde y Juan Cruz Ruiz de Cabañas, trabajaron en la con.

16 Gálvez, Conciencia, 1996, p. 45.

${ }^{17}$ Ibid., p. 51. solidación de Guadalajara al apoyar los proyectos del consulado y de la universidad y fomentar diversas obras públicas. Además su labor no se circunscribió a la ciudad-capital sino que abarcó todo el obispado. La similitud en las conductas de los intendentes y de los obispos, no extrañan a Gálvez Ruiz, ya que estos hombres de fin de siglo responden al perfil del hombre ilustrado.

Para profundizar el papel del clero en las reivindicaciones de carácter regional, contamos con el trabajo de B. Connaughton, Ideología y sociedad en Guadalajara, que ya citamos. Connaughton quiere reconstruir los procesos mentales a través de los cuales la elite eclesiástica neogallega fijó su identidad cultural. El tipo de análi- 
sis que efectúa este autor, se señala ya desde los encabezados de ciertos capítulos: "Las presiones regionales como condicionante ideológico", 18 "La forja de la región", 19 "El clero y el gobierno de Guadalajara en la época colonial: portavoces imperiales de los intereses regionales", 20 "La elaboración de un discurso ideológico regionalista". 21

Su análisis parte del supuesto de que la Iglesia se comprometió con los intereses regionales y marchó al com. pás del cambio. Apoyó los proyectos que propiciaban mejoría y cambio, como el consulado, la universidad y la imprenta y se unió a los proyectos de corte regionalista.

Pero para B. Connaughton: "Guadalajara era una región de definición más bien reciente, donde las reformas borbónicas se habían acoplado a un dina. mismo económico notable". ${ }^{22} \mathrm{Si}$ Connaughton sustenta esto, es porque para él las regiones se definen económicamente, y dada la dependencia que Nueva Galicia tuvo con la Nueva España durante los siglos XVI y XVII:

no había tenido posibilidad alguna de anteponer un espacio económico propio o cuasiautónomo al gran espacio interno del virreinato que regía el consulado de México [...] es cuestionable inclusive si se puede hablar orgánicamente de una región, ya que las fuerzas centrífugas tendían a arrancar de Guadalajara la hegemonía a la cual de. bían aspirar[...]

18 Connaughton, Ideología, 1992, p. 37.

19 Ibid., p. 39.

20 Ibid., p. 67.

21 Ibid., p. 70.

22 Ibid., p. 37.
Por contraste, sin embargo, la tónica del siglo XVIII resultó condicionada por la creciente consolidación de un mercado regional interno basado en la expansiva población de la capital Guadalajara. ${ }^{23}$

Para B. Connaughton, el clero jugó un papel destacado en la formulación ideológica de este regionalismo creciente, en gran medida por el lugar privilegiado que ocupaba, ya que:

Tenía un foro para exponer sus ideas en el púlpito y en la imprenta y era objeto de consulta casi forzada en la forja de nuevas directrices para el desarrollo político y socioeconómico de la zona. ${ }^{24}$

Todos los autores que revisamos coinciden en que las variables que dieron unidad al territorio de Nueva Galicia son múltiples. Sin embargo manejan en forma diferente el factor tiempo.J. P. Berthe plantea el proceso de formación desde una perspectiva de "larga duración" como Braudel lo propone; en cambio la consolidación la sitúan todos nuestros autores dentro de un periodo sobre el cual podríamos decir "la historia se acelera". Pero la mayoría dan preferencia, $y$ posiblemente demasiado peso, a los últimos 25 años del siglo XvIII, sin considerar que su importancia procede de que en esta época encontraron eco viejos reclamos que pudieron finalmente encontrar su cauce.

Lo que podría ayudar a aclarar el problema de la "personalidad regio-

23 Ibid., p. 40.

${ }^{24}$ Ibid., p. 71. 
nal", sería -siguiendo una vez más a J. P. Berthe cuando señala que varios Estados de América Latina independientes coinciden con las audiencias coloniales-, hacernos la pregunta contraria: ¿por qué no surgió en 1821 o entre 1821 y 1846 un Estado indepen. diente de Jalisco? A los historiadores no les gustan esos juegos intelectuales de "simulación" o "Kriegspiel". ${ }^{25}$ Sin embargo podría llevarnos por veredas interesantes.

\section{BIBLIOGRAFIA}

-Altman, Ida y James Lockhart, Provinces of early Mexico, variants of spanish american regional evolution, UNCLA, Latin American Center Publications, Los Ángeles, 1976.

-Berthe, J.P., "Introduction a l'histoire de Guadalajara et sa region", Villes et Régiones en Amérique Latine, IHEAL, 1970, Cahier, núm. 1.

-Calvo, Thomas, Poder, religión y sociedad en la Guadalajara del siglo XVII, CEMCA/Ayuntamiento de Guadalajara, México, 1991.

, "Les réalites du pouvoir aux confins de l'empire", en Les élites locales et l'état, CNRS, París, 1993.

-Connaughton, Brian, Ideología y so-

25 Juego de simulación estratégica practicado en las escuelas de guerra y en los Estados Mayores. ciedad en Guadalajara (1788-1853), CNCA, México, 1992.

-Gálvez Ruiz, Ma. Ángeles, La conciencia regional en Guadalajara y el gobierno de los intendentes. 1786-1800, UNED, México, 1996.

Greenow, Linda, "Spatial dimentions of the credit market in Nueva Galicia, 1721-1820", tesis doctoral, Universidad de Syracuse, 1980.

Credit and socioeconomic change in colonial Mexico. Loans and mortgages in Guadalajara, 1720-1820, Departement of Geography Syracuse 25 University, Syracuse, 1983 (Dellplain Latin American Studies núm. 12).

-Lindley, Richard, Las haciendas y el desarrollo económico. Guadalajara, México en la época de la independencia, FCE, México, 1987.

-Mota Padilla, Matías de la, Historia del reino de la Nueva Galicia en la América septentrional, Universidad de Guadalajara, 1973 (Colección Histórica de Obras Facsimilares).

-Olveda, Jaime, La oligarquía de Guadalajara, CNCA, México, 1991.

-Parry, John N., La Audiencia de la Nueva Galicia en el siglo XVI, traducción de Rafael Diego y Eduardo Williams, El Colegio de Michocán, 1993.

-Serrera, Ramón Ma., Guadalajara ganadera, estudio regional novohispano, 1760-1805, CSIC/Escuela Estudios Hispanoamericanos, Sevilla, 1977.

-Van Young, Eric, La ciudady el campo en el México del siglo XVII. La economía rural de La región de Guadalajara, 1675 1820, FCE, México, 1986. 


\section{SECUENCIA}

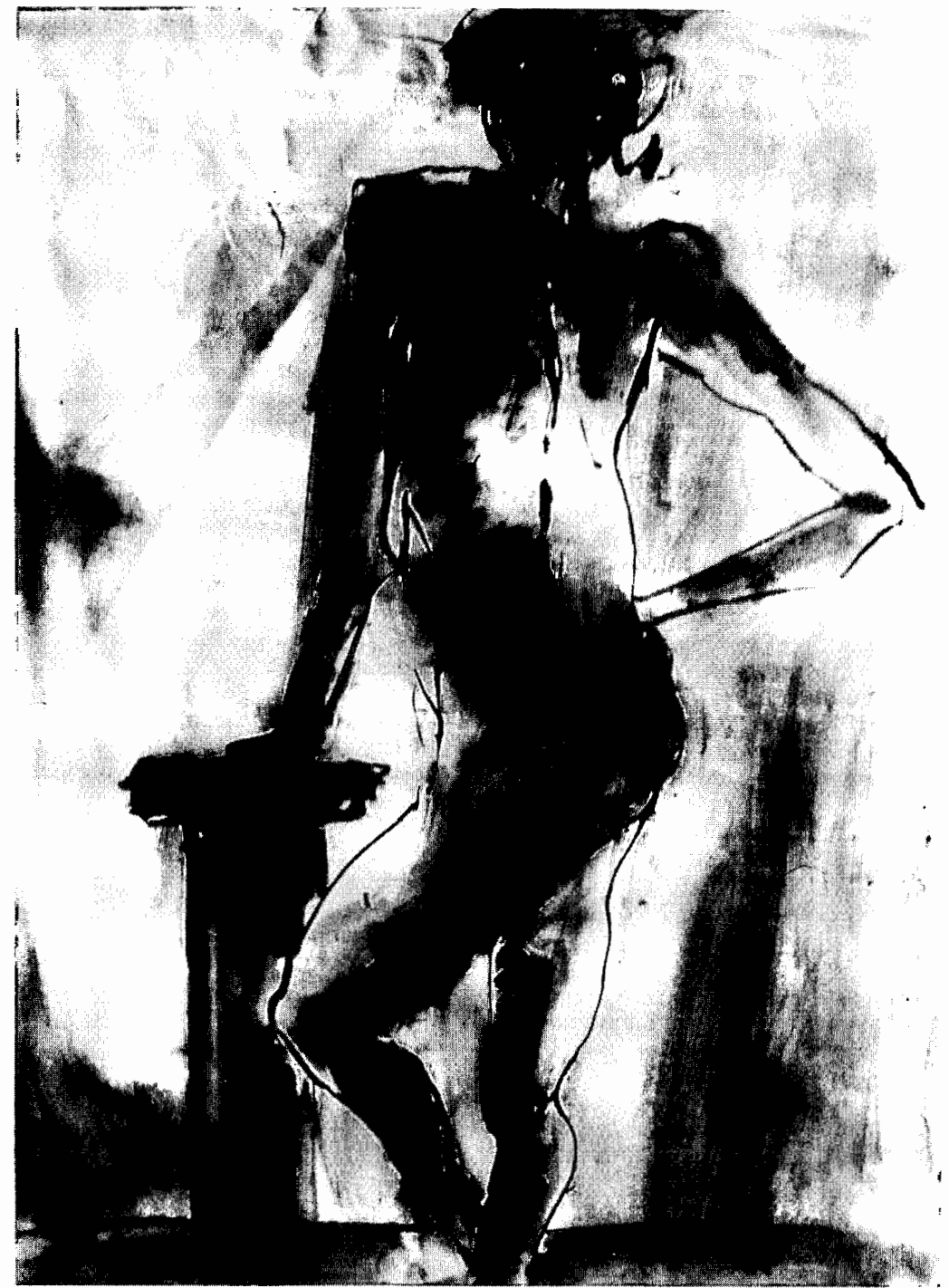

